\title{
Mobile Health Technologies could enhance Public Private Mix for Tuberculosis care in Rural Southwestern Uganda: Qualitative Findings
}

\section{Wilson Tumuhimbise ( $\sim$ twilson@must.ac.ug)}

Mbarara University of Science and Technology Institute of Computer Science https://orcid.org/00000003-1895-9255

\section{Daniel Atwine}

Mbarara University of Science and Technology

\section{Fred Kaggwa}

Mbarara University of Science and Technology

Angella Musiimenta

Mbarara University of Science and Technology

\section{Research Article}

Keywords: mobile health, Tuberculosis, Public private mix

Posted Date: December 1st, 2021

DOI: https://doi.org/10.21203/rs.3.rs-1114770/v1

License: (9) This work is licensed under a Creative Commons Attribution 4.0 International License. Read Full License 


\section{Abstract \\ Background}

Despite some global progress in the implementation of the public-private mix for Tuberculosis care, the engagement of private healthcare providers remains wanting especially in high incidence countries such as Uganda. Although mobile health technologies are low-cost approaches that can enhance Tuberculosis care, there is a dearth of research about their application in fostering public-private mix.

\section{Objective}

To explore the potentials of mobile health technologies in fostering public-private mix for Tuberculosis care in Uganda.

\section{Methods}

This was a qualitative study design that involved in-depth interviews with 13 key informants (private healthcare workers) purposively selected between June and July 2020 due to their active involvement in Tuberculosis care from four private hospitals in Mbarara City. The interviews were transcribed and coded to identify key themes for analysis using content analysis.

\section{Results}

Mobile Health technologies (such as mobile apps, text messages) have the potential to map and link patients from private hospitals to the referral units, support patient medication adherence, notify and report Tuberculosis cases to the Ugandan Ministry of Health, and enhance patient care and monitoring.

\section{Conclusion}

Mobile Health technologies have the potential to revolutionize Tuberculosis care by establishing a centralized pathway for linking the referred patients from private hospitals to public hospitals. Future research should focus on assessing the utilization of mobile health technologies in enhancing access to referral units by presumptive Tuberculosis patients referred from private hospitals in low-resource settings.

\section{Background}

\section{The public-private mix for tuberculosis care}


Tuberculosis (TB) remains one of the top causes of death globally despite its being curable and preventable [1]. Globally, About 10 million people developed TB disease in 2019 of which 1.4 million died [2]. The burden of TB disease in Uganda remains high with an incidence rate of 200 (117-303) per 100,000 [3]. Despite the Human Immunodeficiency Virus (HIV)-Positive TB incidence rate of 78 per 100,000 , Uganda is still ranked among the 30 high TB/HIV burden countries in the world [2]. Developing countries are continuing to struggle towards achieving the 2035 global targets of the End-TB strategy. These include achieving a $95 \%$ decline in deaths due to tuberculosis compared with 2015 , reaching an equivalent $90 \%$ reduction in tuberculosis incidence rate from a projected 110 cases/100 000 in 2015 to 10 cases/100 000 or less by 2035 and that by 2020, no tuberculosis-affected person or family should face catastrophic costs due to tuberculosis care [4]. Intensified research and innovation is one of the pillars for achieving these targets, under which, research to optimize implementation and impact, and promote innovations is a major action. It has been reported that indeed most innovations cannot be translated into effective local action without careful planning and adaptation, and partnership with stakeholders [4]. The private healthcare sector is a key stakeholder in TB care whose engagement needs to be more enhanced by Government-based National Tuberculosis Programs (NTPs). Although previously considered to be better avenues for providing promotive, preventive, curative components of general healthcare [5], government health facilities are continually becoming more overwhelmed by the increased TB disease burden, hence needing supplementation by private health facilities. The public-private mix (PPM) was introduced in 2003 by World Health Organization (WHO) to involve all the relevant healthcare providers (private health facilities) in the provision of TB care and control [6]. There is some evidence that shows that PPM is cost-effective for TB care [7], and increases case detection [8]. From 2010-2019, there has been a significant contribution of PPM which resulted in the increase in the annual number of case notifications (1.8 million cases in 2019) among the high TB burden countries (i.e. Bangladesh, India, Indonesia, Myanmar, Nigeria, Pakistan, and the Philippines) [2]. Despite some global progress in the implementation and expansion of PPM, many private healthcare providers remain unengaged [9]. In Uganda, the engagement of private health providers in TB care and prevention by the Ugandan Ministry of Health/ National Tuberculosis and Leprosy Programme (MOH/NTLP) is minimal despite being a significant contributor of health services, with $47 \%$ of all hospitals in Uganda being private [10]. Although many patients in Uganda access healthcare through private service providers, most private healthcare providers (such as private clinics and hospitals) remain incapacitated to provide TB treatment and care [11]. They mainly screen patients for symptoms and refer patients for better management in government health facilities. This could result in delayed case detection and notification rates.

To address the current gap in PPM for TB care, guidelines for enabling the standardization of PPM for TB were developed in 2018 by Uganda stop TB partnership and the Ugandan Ministry of Health/NTLP [10]. According to this report, the implementation of PPM in Uganda is conducted via a phased approach to enable the engagement of private facilities in TB service provision through training and mentorship, to build the capacity of the participating private health facilities. Progress indicates that a handful of private health practitioners including doctors, nurses, and laboratory personnel from six districts (Mbale, Tororo, Jinja, Iganga, Gulu, and Kitgum) in eastern and Northern Uganda were trained in the implementation of 
PPM activities. In addition to this capacity building, the $\mathrm{MOH}$ in partnership with United States Agency for International Development (USAID) Defeat TB project trained 20 private health practitioners to improve quality TB care to enable private health facilities to improve TB case notification, detection, and treatment in Kampala, Wakiso and Mukono Districts in Central region, Uganda. Although this continuous mentorship and coaching resulted in the engagement of over 150 health facilities in the country [10], facilities and healthcare workers from the southwestern part of the country were left out.

\section{The Potentials Of Mhealth}

Mobile health (mHealth) refers to the use of portable devices with software applications to provide services and manage patient information [12]. These technologies include mobile phones, tablets, and personal digital assistants. The widespread usage of mobile phones globally with the number of subscribers growing exponentially and exceeding even the population size in some countries $[13,14]$ provides opportunities for their use in the health sector. mHealth interventions are less expensive, with the potential to reach a wide client base and the hard-to-reach groups $[15,16]$. The adoption and integration of mHealth in the routine operations of the health sector have been slow compared to other sectors like banking, airline, and insurance [17]. There is some evidence that mHealth technologies can enhance PPM through TB screening, case notification, treatment adherence, data collection and management, patient referral, and follow-up [18] and promote the adoption of health behaviors including HIV/AIDS prevention $[19,20]$ and prenatal service utilization [21-23].

The use of mHealth technologies (e.g. smartphone apps, text messages, wearable devices) in supporting TB care is highly recommended by the WHO as emerging opportunities that can close the gaps in TB care [24]. These technologies can potentially enhance the identification of lost follow-ups [25] among TB patients, support TB case identification, diagnosis, notification, reporting, and monitor drug adherence [26]. Although the application of mHealth in TB care and management particularly in developing countries is still in its infancy, the modest existing studies show some potential. For instance, mHealth technologies (e.g., SMS texts and real-time medication adherence monitors) can improve TB medication adherence through reminders [27], foster the provision of social support (e.g. money sent on mobile phones for transport, phone calls for emotional support) to TB patients [28].

mHealth technologies are low-cost applications that are sensitive to both the private sector ways of working and the expectations of the NTPs [9] with the ability to produce tremendous results in facilitating the engagement of the private hospitals. However, there is a dearth of research about their application in fostering PPM. This knowledge would practically inform the future development of mHealth interventions for engaging private health facilities in TB care. Yet the use of such technologies can potentially address the current highlighted by Khan and colleagues [29]- weak patient referral systems, lack of appropriate patient tracking and support systems, and the lack of the ability to cross-link and identify patients who move between private and public sectors, which continue to cripple the performance of TB control 
programs. The purpose of this study was to explore the potentials of mHealth in fostering PPM in Uganda.

\section{Methods}

\section{Study design and setting}

We conducted a cross-sectional study with a qualitative research approach that involved in-depth semistructured interviews with healthcare workers (medical doctors and nurses). The methodology for this study has been described previously [30]. Briefly, the participants were recruited from three private forprofit (PFP) and one private not-for-profit (PNFP) hospital in Mbarara City, in South-Western Uganda.

1. Hospital 1: a private for-profit hospital that provides general medical consultation services to over 2,600 patients that attend the Outpatient department (OPD) and over 60 TB patients annually. The hospital screens, diagnoses and refers TB patients to the Mbarara regional referral Hospital (MRRH) (Number/cadre of healthcare workers involved in TB-related care: four medical doctors and six nursing officers).

2. Hospital 2: a private not-for-profit faith-based hospital with an OPD that serves over 27,764 patients and over 100 TB patients annually and offers general medical treatment and Antiretroviral treatment (ART) services. The hospital screens, diagnoses and manages TB patients (Number/cadre of healthcare workers involved in TB-related care: four medical doctors and eight nursing officers).

3. Hospital 3: a private for-profit hospital providing general medical consultation services to over 13,835 patients in the OPD and over 70 TB patients annually and has an ART clinic where HIV patients receive their treatment and care. The hospital screens, diagnoses and manages TB patients (Number/cadre of healthcare workers involved in TB-related care: five medical doctors and 10 nursing officers).

4. Hospital 4: a private for-profit hospital providing general medical consultation services to over 1500 patients and over 40 TB patients annually. The hospital screens, diagnoses, and refers TB patients to MRRH (Number/cadre of healthcare workers involved in TB related care: four medical doctors and six nursing officers)

These facilities were purposively selected for having relatively large medical establishments, a large number of health care workers, being involved in TB case detection, and being at the center of handling a large number of outpatients in Mbarara City, which increases the likelihood of detection of presumptive TB cases. After performing symptom-based screening of patients for TB, all hospitals refer the presumptive TB patients to Mbarara Regional Referral hospital which is the only NTP facility that manages Tuberculosis in Western Uganda.

\section{Selection of study participants}


Key informants from four private hospitals in Mbarara City were purposively selected based on their current or previous involvement in TB-related activities such as case identification, referral, and treatment, as well as their willingness to participate in the study. A total of 13 healthcare workers $(11$ medical doctors and two nurses) were recruited (Table 1 below). Specifically, participants were included based on the following inclusion criteria: a) employed medical doctor/nurse in a private hospital setting, b) have experience in TB-related activities, c) willing and able to give Informed consent.

\section{Data collection}

Between June and July 2020, a semi-structured interview guide was developed in English, pre-tested to ensure data reliability, validity and accuracy. The researcher (WT) who has experience and training in qualitative research and research ethics, conducted semi-structured in-depth interviews with the medical doctors and nurses from the identified study sites. The interviews involved engaging the health workers to tell their stories as prompted by the investigator. Each interview lasted between 30 and 40 minutes and was carried out in a private convenient place preferred by the respondents. Interviews were recorded digitally with participants' consent and transcribed thereafter. Questions about the perceived benefits and barriers of mHealth in fostering PPM were elicited. Following each conducted interview, the transcripts were reviewed for quality assurance, clarity, and detail by authors AM, AD and FK. A short survey was then administered to the medical doctors and nurses to gather their social demographic details.

\section{Analysis}

Through the initial review of transcripts, researcher WT developed an initial coding scheme which was reviewed and discussed by authors AM and FK. The inductive content analysis approach [31] was used to generate themes and subthemes from the codebook. Categories about the potentials of mHealth (e.g. Referral, supporting medication adherence, TB case notification) in fostering PPM in TB care were extracted from the transcripts. The codebook was reviewed by AM to ensure that the relevant content was extracted. WT assembled the codebook following an iterative approach that included category construction of codes (namely: Mapping and linking referred patients to the referral points, supporting patient care and medication adherence, and Tuberculosis cases notification and reporting) to represent the content, writing the operational definitions, elaborations and illustrative quotes. Intercooled STATA (Statacorp 2013 Stata Statistical Software Release 13; StataCorp, College Station, TX, USA) was used to describe study participants' characteristics.

\section{Results}

Thirteen healthcare workers (key informants) composed of 11 medical doctors (85\%) and two nurses (15\%) from four private hospitals in Mbarara City participated in this study. The majority of the participants $8(62 \%)$ were male, with a median age of 29 years, the majority were recruited from private for-profit private hospitals (85\%) and with 5 or fewer years of medical practice; as indicated in Table 1 below 
Table 1

Participants' Demographics 


\begin{tabular}{|c|c|}
\hline \multirow[t]{2}{*}{ Baseline characteristic } & \multirow{2}{*}{$\begin{array}{l}\text { Participants } \\
n\end{array}$} \\
\hline & \\
\hline Median Age (IQR) & $29(28-31)$ \\
\hline \multicolumn{2}{|l|}{ Gender } \\
\hline Female & 5 \\
\hline Male & 8 \\
\hline \multicolumn{2}{|l|}{ Marital status } \\
\hline Single & 5 \\
\hline Married/partnered & 8 \\
\hline \multicolumn{2}{|l|}{ Highest educational level } \\
\hline Certificate & 1 \\
\hline Diploma & 1 \\
\hline Degree & 7 \\
\hline Masters & 3 \\
\hline PhD & 1 \\
\hline \multicolumn{2}{|l|}{ Designation } \\
\hline Nurse & 2 \\
\hline Doctor & 11 \\
\hline \multicolumn{2}{|c|}{ Number of years in general medical practice } \\
\hline $1-3$ & 7 \\
\hline $4-5$ & 2 \\
\hline$>5$ & 4 \\
\hline \multicolumn{2}{|c|}{ TB services provided by healthcare workers } \\
\hline Screen and manage ${ }^{a}$ & 5 \\
\hline Screen and refer ${ }^{b}$ & 8 \\
\hline \multicolumn{2}{|c|}{ Note. IQR - interquartile range. } \\
\hline \multicolumn{2}{|c|}{ a Participants able to both screen and manage TB cases } \\
\hline b Participants able to bo & umptive case \\
\hline
\end{tabular}




\section{Interview results}

The study identified several potentials of mHealth in fostering PPM in TB care. Participants felt that $\mathrm{mHealth}$ has the potential to map and link the referred patients to the referral units, support patient care and medication adherence, and notify and report TB cases.

\section{Mapping and linking referred patients to the referral points}

mHealth was reported to have the potential to map and link presumptive TB patients that have been referred from private hospitals to the hospital facilities where they have been referred for TB services. This involves the identification of presumptive TB patients and ensuring that they have been linked or connected to the nearest clinics that provide the required TB services. One medical doctor from Hospital 1 reported that "mHealth can help in mapping and linking the patients to the nearest health facility that offers the TB services". In addition, mHealth technologies were perceived to be useful in informing the referral centers about the details of an individual patient that needs to be attended to, which could potentially result in faster services. One participant from Hospital 4 described,

If I get a presumptive TB patient at the private facility, through the mobile app, I can inform the TB clinic at the public hospital which is licensed to provide TB services that on a certain day, a patient from a certain hospital is coming, it will result in faster care on the presumptive TB case, [Medical Doctor, Hospital 4].

\section{Supporting patient care and medication adherence}

Participants perceived mHealth as a tool for enhancing coordinated patient care and patient monitoring through facilitating communication between patients and healthcare workers. A medical doctor from Hospital 1 reported that

I think it would help if someone had an app on their phone installed that would keep the patient and the health care providers in touch, which would ensure better adherence to the drugs among patients, and better follow-up, [Medical Doctor, Hospital 1].

The communication between patients and patients would enable patients to adhere to their medication via SMS reminders, and be encouraged to take their medication on time, and attend their clinical appointments as scheduled. A medical doctor from Hospital 4 reported that "I think mHealth can help in monitoring patients to take their medication on time, and also help them know when to refill their drugs and also reminders of when to come back to the clinic".

\section{Tuberculosis cases notification and reporting}

The participants also highlighted that mHealth can enable timely reporting and notifying of TB cases to the ministry of health on what is happening in the private hospitals. This would be through enhanced remote uploading of daily, weekly reports about activities to the government. This would help in reducing 
time to notify TB cases between different health centers and updating the national database about the number of cases. One participant from Hospital 3 reported that

$m$ Health can help in reporting and giving timely reports. You can design an app where we can upload the work we are doing weekly so that we constantly update the government on the work that is going on. If there is a case that I have diagnosed, I can easily upload patients' details in the mobile application, which can quickly update the number of cases at the national level from both the public and private hospitals, [Medical Doctor, Hospital 3].

\section{Concerns: Lack of knowledge to use the technology}

Some participants expressed concerns about the lack of knowledge among the health workers to use the developed mobile applications which might hinder the operation and implementation of the technology due to abandonment by the intended user. One medical doctor from Hospital 2 reported that "Some health workers may not know how to use the app and in the long run may abandon it".

\section{Concerns: Poor internet connection}

Participants also raised the concern of poor internet connection (e.g., slow connections or lack of internet) which might affect the usability of mHealth interventions that rely entirely on the internet. One participant from Hospital 3 "If the app uses the internet, in some places, there is poor network connections, which might affect the operation of the intervention".

\section{Discussion}

Our study explored the potentials of mHealth in fostering PPM in Uganda. Overall, key informants discussed the potentials of mHealth technologies in fostering PPM in TB care. mHealth technologies (such as mobile apps, text messages) have the potential to map and link patients from private hospitals to the referral units, support patient care and medication adherence, as well as notify and report TB cases to the Ugandan Ministry of Health.

\section{Meaning Of The Study And Comparison With Other Studies}

The potential of mHealth to map and link referred patients to the referral points could eliminate the risk of loss of patients along the pathway of care but could also result in enhanced patient care and monitoring. Through such mHealth interventions, the proportion of patients that reach their expected referral points and who get started on medication on time could increase, with subsequent reductions in diagnostic and drug initiation delays. Most of the private hospitals in Uganda are not accredited to provide TB services, this limits their efforts and engagement in TB care despite being at the center of receiving a majority (48.4\%) of the population who seek healthcare compared to the government (public) health facilities (34\%) [32]. Therefore, as a common practice, most of these private hospitals refer presumptive TB patients to public hospitals [33]. However, the lack of mechanisms for following up these patients in 
private hospitals [30] still stifles TB control efforts. In this regard, the adoption of the current ICT technologies like smartphones, mobile apps (mHealth) as a mechanism for following up these patients after referral could ensure easier patient tracking and successful referral processes from private to public health facilities. For example, the use of telephone calls as a measure for following up the referred TB patients from private facilities to the peripheral units has been reported in Cambodia and resulted in increased retention of the referred TB patients [34]. In India the utilization of a smartphone application by rural private healthcare practitioners to refer patients to TB microscopy Centre located at public health facilities resulted in an increased number of referrals, reduced time taken for diagnosis, and treatment initiation [35]. However, on the other hand, the implementation and utilization of mHealth in fostering PPM in Africa remain unknown, yet most countries in Africa still grapple with a high number of TB cases (25\% of the global TB cases come from Africa) [2]. Understanding how mHealth is utilized to engage the private sector in TB care especially in African settings has the potential to ensure better TB disease outcomes in Africa.

The potential of mHealth in supporting patient care and medication adherence among TB patients could enhance timely in-take of medication and the attendance of hospital appointments for pill refills. Particularly in our study, participants reported that mHealth technology would enable healthcare workers to monitor patients thus enabling adherence to medication and attendance of hospital appointments. mHealth provides quick access to information for both patients and healthcare workers and offers a qualitative and quantitative interaction between the healthcare providers and the patients [23]. Several studies utilizing mHealth technologies for supporting medication adherence among both TB and HIV patients have been conducted in Uganda $[19,27]$ and were found to be acceptable and feasible. A randomized control trial [36] that was conducted in Karachi Pakistan utilized two-way short message service (SMS) reminders to encourage newly diagnosed patients from public and private health facilities to take their medication. However, the utilization of mHealth technologies between the public and private health facilities in Uganda for TB care is unknown. This leaves a research gap and calls for a special investigation on how mHealth technologies can be utilized for enhancing PPM in TB care.

Our study findings report that mHealth interventions could also enhance quick and timely notification of TB cases from the private health facilities to national TB programs to ensure better planning of TB management services. Notifying cases is key in enhancing the tracking and management of the patients. TB case notification rate in Uganda is mostly done by public hospitals (86\%), while private for-profit facilities notify only $1 \%$ [37]. The report shows that most of the cases are still missed where about 30,000 people with TB symptoms were neither notified nor diagnosed in 2018/19. This implies a need for establishing mechanisms for ensuring that all cases are notified. mHealth could play a role in improving notification of every diagnosed case as shown in the literature. According to a scoping review by Tumuhimbise and Musiimenta, mHealth interventions like mobile applications have been utilized in the notification of TB cases in Vietnam India, Indonesia, Pakistan, and Vietnam [18]. A referral and reporting back mobile phone application was found to be acceptable and feasible for notifying TB cases among the private practitioners in Indonesia [38]. Although mHealth has been utilized in the notification of TB cases elsewhere, its experience among healthcare workers in settings like Uganda where healthcare 
workers might be overwhelmed with a large number of clients (high patient-doctor ratio) and fail to find time for real-time use of the application to notify cases; this is being explored in our ongoing research investigation.

In this study, participants raised concerns about the lack of knowledge to use the developed mobile applications. User involvement during intervention development may play a role in acquainting system end-users with the intervention which in the long run will facilitate the acceptability of the developed application among users [39]. This approach helps the developers to understand user behaviors, requirements, and skills at each phase of the design [40]. Training users on how to use the application before implementation may improve usability and address this concern. Users who are not well trained to use find it hard to use the technology [41]. Therefore, system implementers should ensure conducting initial training sessions before rolling out the interventions. In addition, developers should develop interventions that are easy to use [18] which are intended to ease the work of healthcare workers.

The reported poor internet connectivity or lack of internet access especially for interventions that entirely run on the internet or require the internet to function can hinder the usability of the application and result in abandonment of the interventions. mHealth interventions that entirely rely on the internet to operate, require stable internet connections to ensure thorough operability. The jurisdiction of the cost burden of internet packages for running these internet-based interventions must be clearly defined lest the cost burden on the users can't be sustained in the long run. mHealth interventions should at all costs remove the cost burden from the users if the successful implementation is to be ensured. Considering the development of standalone interventions or applications that do not require the internet to operate can address this concern.

The general implementation and coverage of PPM for TB in Uganda require deliberate efforts to ensure that all private health facilities are involved in TB care if success and better health outcomes are to be realized. Engaging and empowering the private sector in TB disease management is crucial in enhancing the reduction of unnecessary deaths and sufferings due to substandard diagnosis, inappropriate treatment, and care that occur in the private sector, thus reduction in catastrophic costs [42]. Successful TB control efforts cannot be achieved globally if the private providers are not brought on board to tackle TB disease.

\section{Strength of the study}

This study has several strengths; first, it identifies key insights about the potentials of mHealth technologies from the front line private medical healthcare workers in a low resource setting which provides an evidence base for the development of future interventions in similar settings. Secondly, to the best of our knowledge, this study is the first to report pre-intervention perceptions of healthcare workers from the private hospitals about the potentials of mHealth technologies for fostering PPM in TB care. Third, our findings are important for understanding the optimal design of future mHealth interventions to facilitate sustainable PPM in TB care. 


\section{Limitations of the study}

Our study is not without limitations that should be considered during the interpretation of our research findings. First, being a formative study, the investigator solicited the perceptions of private healthcare workers about using mHealth in TB care before they could use the intervention in real life. The results, therefore, do not document the practical real-life experiences of utilizing the mHealth intervention in TB care. Second, our study employed a purposive sampling approach for recruiting key informants, which has the potential for selection bias. Lastly, this study was conducted in private hospitals in an urban setting; this could limit the generalizability of our findings in rural settings, where private hospital operations and practices might be different.

\section{Implications for policy and practice makers}

The utilization of mHealth in TB care among private hospitals in Uganda is lacking. This limits the realization of mHealth benefits in the achievement of better TB treatment outcomes and the realization of PPM. Our findings imply that mHealth has the potential to revolutionize TB care by establishing a centralized pathway for linking the referred patients from private to public hospitals and ensuring that they are not lost along the TB care pathway, have been diagnosed, and started on medication. Our study implies that mHealth can be a cost-effective solution for enhancing the full integration and engagement of the private healthcare facilities in TB care thus the achievement of PPM.

The implementation of the mHealth interventions requires training of users to equip them with skills to enable intervention acceptability and usability. mHealth interventions that entirely rely on the internet to operate, require stable internet connections to ensure thorough operability. Standalone applications that do not require the internet to operate can offer better alternatives. mHealth interventions should at all costs remove the cost burden from the users if the successful implementation is to be ensured.

Our findings recognize that there is a need for future researchers to assess the utilization of mHealth for following up patients that have been referred from the private hospitals in African settings to ascertain its effectiveness on TB care outcomes and its contribution to the PPM effort for TB care.

\section{Unanswered questions and future research}

Exploring the real-life experiences of how mHealth can be used to foster the establishment of PPM in rural Uganda is key in informing policy formulation to address TB care outcomes.

\section{Conclusion}

This study showed that mHealth could provide acceptable and feasible approaches to map and link TB patients to the referral units, enhance patient care and medication adherence and notify TB cases. Understanding how mHealth technologies are perceived by private healthcare workers is key in informing the development of interventions that are acceptable and feasible and effective in enhancing the engagement of private hospitals in TB care. Findings from this formative study are informing the 
development of an m-Health intervention to enhance the engagement of private hospitals in TB care, which the authors are currently working on. The utilization of mHealth could offer a cost-effective solution in enhancing the implementation of the PPM. The Private hospitals, policymakers, researchers, and developers need to bear in mind that the utilization of mobile health technologies not only improves the quality of care among the presumptive TB patients but also could enhance better treatment outcomes like early diagnosis and drug initiation thus limiting the risk of infection at both the community and household level.

\section{Abbreviations}

WHO: World Health Organization; TB: Tuberculosis; PPM: public private mix; HIV: Human Immunodeficiency virus; SMS: Short Message Service; USAID: United States Agency for International Development; NTP: National Tuberculosis Program

\section{Declarations}

\section{Ethics approval and consent to participate}

Official permission was sought and obtained from the participating private hospitals where the study was conducted. Ethical approval was obtained from Mbarara University Ethics Committee- MUREC (Protocol number: 32/03-20) and the Uganda National Council of Science and Technology - UNCST (Registration number: HS963ES). All participants provided written consent before participating in the study.

\section{Consent for publication}

Not applicable

\section{Availability of data and materials}

The datasets used and/or analysed during the current study are available from the corresponding author on reasonable request

\section{Competing interests}

The authors declare that they have no competing interests

\section{Funding}

Research reported in this publication was supported by the Eunice Kennedy Shriver National Institute of Child Health \& Human Development of the National Institutes of Health under Award Number R21HD107985. The content is solely the responsibility of the authors and does not necessarily represent the official views of the National Institutes of Health.

\section{Authors' contributions}


Conceptualization: WT, AM. Data curation: WT. Formal analysis: WT, AM. Funding acquisition: AM. Methodology: WT, AD. Supervision: AD, FK, AM. Literature Review: WT. Writing original draft: WT. Writing review \& editing: WT, AM, AD, FK. All authors read and approved the final manuscript.

\section{Acknowledgements}

This work is part of a PhD research approved by Mbarara University Research Ethics Committee MUREC (Protocol number: 32/03-20), and the Uganda National Council of Science and Technology UNCST (Registration number: HS963ES) supervised by Dr. Angella Musiimenta (PhD), Dr. Daniel Atwine $(\mathrm{PhD})$, and Dr. Fred Kaggwa (PhD), in the Faculty of Computing and Informatics (FCl) at Mbarara University of Science and Technology, who guided the investigator throughout the entire process and reviewed the manuscript for correctness and validity.

Dr. Musiimenta is also supported by the Fogarty International Center of the National Institutes of Health K43TW010388-05S1, R21HD107985, the SPIDER (Swedish Program for ICT in Developing and Emerging Regions (Dnr: SU-323-0060-21) and the German Federal Ministry of Education and Research (01DG21014). I would like to acknowledge Associate Professor Luke Davis (Yale School of Medicine) for the support in conceptualizing the research idea.

\section{Authors' information (optional)}

\section{References}

1. WHO. Public-Private Mix for TB Care and Prevention. 2017.

2. WHO. Global tuberculosis report 2020. Glob Tuberc Rep. 2020;2020.

3. WHO. Tuberculosis profile: Uganda 2020 [cited 2021 21st July]. Available from: https://worldhealthorg.shinyapps.io/tb_profiles/? _inputs_\&entity_type=\%22country $\% 22 \& l a n=\% 22$ EN $\% 22 \&$ iso2=\%22UG $\% 22$.

4. WHO. The End TB Strategy. Global strategy and targets for tuberculosis prevention, care and control after 2015. World Health Organization. 2014.

5. WHO. Involving private practitioners in tuberculosis control: issues, interventions, and emerging policy framework. In Involving private practitioners in tuberculosis control: issues, interventions, and emerging policy framework. 2001.

6. Uplekar M. Involving private health care providers in delivery of TB care: global strategy. Tuberculosis. 2003;83(1-3):156-64. https://doi.org/10.1016/s1472-9792(02)00073-2

7. Anand T, Babu R, Jacob AG, Sagili K, Chadha SS. Enhancing the role of private practitioners in tuberculosis prevention and care activities in India. Lung India: Official Organ of Indian Chest Society. 2017;34(6):538. https://doi.org/10.4103/0970-2113.217577

8. Asuquo AE, Pokam BDT, Ibeneme E, Ekpereonne E, Obot V, Asuquo P. A public-private partnership to reduce tuberculosis burden in Akwa Ibom State, Nigeria. International journal of mycobacteriology. 
2015;4(2):143-50. https://doi.org/10.1016/j.ijmyco.2015.04.002

9. Uplekar M. Public-private mix for tuberculosis care and prevention. What progress? What prospects? The International Journal of Tuberculosis and Lung Disease. 2016;20(11):1424-9. https://doi.org/10.5588/ijtld.15.0536

10. MOH. A Bulletin of the National Tuberculosis and Leprosy Program. Quarterly Bulletin. 2018.

11. Wynne A, Richter S, Banura L, Kipp W. Challenges in tuberculosis care in Western Uganda: health care worker and patient perspectives. International Journal of Africa Nursing Sciences. 2014;1:6-10. https://doi.org/10.1016/j.ijans.2014.05.001

12. Källander K, Tibenderana JK, Akpogheneta OJ, Strachan DL, Hill Z, ten Asbroek AH, et al. Mobile health (mHealth) approaches and lessons for increased performance and retention of community health workers in low-and middle-income countries: a review. Journal of medical Internet research. 2013;15(1):e17. https://doi.org/10.2196/jmir.2130

13. Tamrat T, Kachnowski S. Special delivery: an analysis of mHealth in maternal and newborn health programs and their outcomes around the world. Maternal and child health journal. 2012;16(5):1092101. https://doi.org/10.1007/s10995-011-0836-3

14. Aranda-Jan CB, Mohutsiwa-Dibe N, Loukanova S. Systematic review on what works, what does not work and why of implementation of mobile health (mHealth) projects in Africa. BMC public health. 2014;14(1):1-15. https://doi.org/10.1186/1471-2458-14-188

15. Evans C, Turner K, Suggs L, Occa A, Juma A, Blake H. Developing a mHealth intervention to promote uptake of HIV testing among African communities in the UK: a qualitative study. BMC Public Health. 2016;16(1):1-16. https://doi.org/10.1186/s12889-016-3278-4

16. Déglise $C$, Suggs LS, Odermatt P. SMS for disease control in developing countries: a systematic review of mobile health applications. Journal of Telemedicine and Telecare. 2012;18(5):273-81. https://doi.org/10.1258/jtt.2012.110810

17. Bhattacharya S, Kumar A, Kaushal V, Singh A. Applications of m-Health and e-Health in Public Health Sector: the challenges and opportunities. International Journal of Medicine and Public Health. 2018;8(2). https://doi.org/10.5530/ijmedph.2018.2.12

18. Tumuhimbise W, Musiimenta A. A review of mobile health interventions for public private mix in tuberculosis care. Internet Interventions. 2021:100417. https://doi.org/10.1016/j.invent.2021.100417

19. Musiimenta A, Atukunda EC, Tumuhimbise W, Pisarski EE, Tam M, Wyatt MA, et al. Acceptability and feasibility of real-time antiretroviral therapy adherence interventions in rural Uganda: mixed-method pilot randomized controlled trial. JMIR mHealth and uHealth. 2018;6(5):e9031. https://doi.org/10.2196/mhealth.9031

20. Musiimenta A. Information technology-mediated issues in sexual health and HIV/AIDS education. The Electronic Journal of Information Systems in Developing Countries. 2012;53(1):1-12. https://doi.org/10.1002/j.1681-4835.2012.tb00376.x

21. Musiimenta A, Tumuhimbise W, Pinkwart N, Katusiime J, Mugyenyi G, Atukunda EC. A mobile phonebased multimedia intervention to support maternal health is acceptable and feasible among illiterate 
pregnant women in Uganda: Qualitative findings from a pilot randomized controlled trial. Digital Health. 2021;7:2055207620986296. https://doi.org/10.1177/2055207620986296

22. Musiimenta A, Tumuhimbise W, Mugyenyi G, Katusiime J, Atukunda EC, Pinkwart N. Mobile phonebased multimedia application could improve maternal health in rural southwestern Uganda: mixed methods study. Online Journal of Public Health Informatics. 2020;12(1). https://doi.org/10.5210/ojphi.v12i1.10557

23. Tumuhimbise W, Atukunda EC, Ayebaza S, Katusiime J, Mugyenyi G, Pinkwart N, et al. Maternal health-related barriers and the potentials of mobile health technologies: qualitative findings from a pilot randomized controlled trial in rural southwestern Uganda. Journal of family medicine and primary care. 2020;9(7):3657. https://doi.org/10.4103/jfmpc.jfmpc_281_20

24. WHO. Global tuberculosis report 2019. 2019.

25. WHO. Public-private mix for TB prevention and care: a roadmap. World Health Organization, 2018.

26. WHO. Handbook for the use of digital technologies to support tuberculosis medication adherence: World Health Organization; 2017.

27. Musiimenta A, Tumuhimbise W, Mugaba AT, Muzoora C, Armstrong-Hough M, Bangsberg D, et al. Digital monitoring technologies could enhance tuberculosis medication adherence in Uganda: Mixed methods study. Journal of clinical tuberculosis and other mycobacterial diseases. 2019;17:100119. https://doi.org/10.1016/j.jctube.2019.100119

28. Musiimenta A, Tumuhimbise W, Atukunda EC, Mugaba AT, Muzoora C, Armstrong-Hough M, et al. Mobile Health Technologies May Be Acceptable Tools for Providing Social Support to Tuberculosis Patients in Rural Uganda: A Parallel Mixed-Method Study. Tuberculosis research and treatment. 2020;2020. https://doi.org/10.1155/2020/7401045

29. Khan BJ, Kumar AMV, Stewart A, Khan NM, Selvaraj K, Fatima R, et al. Alarming rates of attrition among tuberculosis patients in public-private facilities in Lahore, Pakistan. Public health action. 2017;7(2):127-33. https://doi.org/10.5588/pha.17.0001

30. Tumuhimbise W, Musiimenta A. Barriers and Motivators of Private Hospitals' Engagement in Tuberculosis Care in Uganda. Global Implementation Research and Applications. 2021:1-12. https://doi.org/10.1007/s43477-021-00030-3

31. Hsieh H-F, Shannon SE. Three approaches to qualitative content analysis. Qualitative health research. 2005;15(9):1277-88. https://doi.org/10.1177/1049732305276687

32. Uganda Bureau of Statistics. Uganda National Household Survey 2016/2017 Report. UBOS. 2017.

33. O'hanlon B, Nakyanzi A, Musembi V, Busulwa I, Husband R, Okumu R, et al. Exploring partnership opportunities to achieve universal health access: 2016 Uganda Private Sector Assessment in Health. 2017.

34. Choun K, Achanta S, Naik B, Tripathy JP, Thai S, Lorent N, et al. Using mobile phones to ensure that referred tuberculosis patients reach their treatment facilities: a call that makes a difference. BMC Health Services Research. 2017;17(1):1-7. https://doi.org/10.1186/s12913-017-2511-x 
35. Chadha S, Trivedi A, Nagaraja S, Sagili K. Using mHealth to enhance TB referrals in a tribal district of India. Public health action. 2017;7(2):123-6. https://doi.org/10.5588/pha.16.0080

36. Mohammed S, Glennerster R, Khan AJ. Impact of a daily SMS medication reminder system on tuberculosis treatment outcomes: a randomized controlled trial. PloS one. 2016;11(11):e0162944. https://doi.org/10.1371/journal.pone.0162944

37. Ministry of Health. National Strategic Plan for Tuberculosis and Leprosy Control 2020/21 - 2024/25. Ministry of Health. 2020.

38. Lestari BW, Arisanti N, Siregar AY, Sihaloho ED, Budiman G, Hill PC, et al. Feasibility study of strengthening the public-private partnership for tuberculosis case detection in Bandung City, Indonesia. BMC research notes. 2017;10(1):1-6. https://doi.org/10.1186/s13104-017-2701-y

39. Goold P, Bustard S, Ferguson E, Carlin E, Neal K, Bowman C. Pilot study in the development of an interactive multimedia learning environment for sexual health interventions: a focus group approach. Health education research. 2006;21(1):15-25. https://doi.org/10.1093/her/cyh040

40. Wever R, Van Kuijk J, Boks C. User-centred design for sustainable behaviour. International journal of sustainable engineering. 2008;1(1):9-20. https://doi.org/10.1080/19397030802166205

41. Gurupur VP, Wan TT. Challenges in implementing mHealth interventions: a technical perspective. Mhealth. 2017;3. https://doi.org/10.21037/mhealth.2017.07.05

42. WHO. Engaging private health care providers in TB care and prevention: a landscape analysis. World Health Organization, 2018. 